\title{
ON SOME INEQUALITIES FOR SPACE HARMONIC FUNCTIONS
}

\author{
M. A. LAVRENTIEV and M. M. LAVRENTIEV
}

Let $f(z)$ be an analytic function, regular in a bounded domain $D$ with a sufficiently smooth boundary $\Gamma$, and continuous in $\bar{D}=D \cup \Gamma$. Let $A$ be a Lebesgue measurable set on $\Gamma, A \subset \Gamma$.

Denote

$$
\sup _{z \in \Gamma}|f(z)|=M, \quad \sup _{z \in A}|f(z)|=\varepsilon .
$$

Then the following inequality holds

$$
|f(z)| \leqq M^{1-\omega(z)} \cdot \varepsilon^{\omega(z)},
$$

where $\omega(z)$ is the harmonic measure of the set $A$ in the domain $D$ with respect to the point $z$. In addition,

$$
a(z) \mu(A) \leqq \omega(z) \leqq b(z) \mu(A),
$$

where $\mu(A)$ is the Lebesgue measure of $A$, and $a(z), b(z)$ are functions independent of $A$. (See [1].)

In spaces having more than two dimensions, one of the natural analogues for analytic functions of complex variable are vector gradients of harmonic functions. Inequalities of type (1) for gradients of space harmonic functions have been established for sets $A$ containing open subsets $[2,3,4]$, and also for sets invariant under a rotation group [5]. The purpose of this article is to establish inequalities of type (1) for harmonic functions in space when sets $A$ with positive plane measure are located on surfaces lying inside the domain of regularity.

The proof of some inequalities in this article is based on a theorem from Chapter III of [4]. Let us formulate this theorem in a slightly modified form.

The or e m 1. Let $f\left(\zeta_{1}, \zeta_{2}\right)$ be a function of two complex variables $\zeta_{j}=\xi_{j}+i \eta_{j}(j=1,2)$ analytic in the bicylinder

$$
\xi_{1}^{2}+\xi_{2}^{2}<R^{2}, \quad\left|\eta_{j}\right|<H, \quad j=1,2,
$$


and continuous in the closed domain

$$
\xi_{1}^{2}+\xi_{2}^{2} \leqq R^{2}, \quad\left|\eta_{j}\right| \leqq H .
$$

Let $A$ be a set with positive Lebesgue measure $\mu(A)$ which lies in the disk

of the real plane.

$$
\xi_{1}^{2}+\xi_{2}^{2} \leqq r^{2}<R^{2}
$$

If the function $f\left(\zeta_{1}, \zeta_{2}\right)$ satisfies the inequalities

$$
\begin{gathered}
\left|f\left(\xi_{1}, \xi_{2}\right)\right| \leqq \varepsilon, \quad\left(\xi_{1}, \xi_{2}\right) \in A, \\
\left|f\left(\zeta_{1}, \zeta_{2}\right)\right| \leqq M,
\end{gathered}
$$

then the following inequality holds

$$
\left|f\left(\zeta_{1}, \zeta_{2}\right)\right| \leqq M^{1-\omega\left(\zeta_{1}, \zeta_{2}\right)} \cdot \varepsilon^{\omega\left(\zeta_{1}, \zeta_{2}\right)},
$$

where $\omega\left(\zeta_{1}, \zeta_{2}\right)$ is a real function not depending on $M, \varepsilon$, with the following properties:

1. $0 \leqq \omega\left(\zeta_{1}, \zeta_{2}\right) \leqq 1$,

2. $\omega\left(\xi_{1}, \xi_{2}\right)=1, \quad\left(\xi_{1}, \xi_{2}\right) \in A$,

3. $a\left(\zeta_{1}, \zeta_{2}\right) \mu(A) \leqq \omega\left(\zeta_{1}, \zeta_{2}\right), \quad \xi_{1}^{2}+\xi_{2}^{2}<R^{2}, \quad\left|\eta_{j}\right|<H$.

Here the function $a\left(\zeta_{1}, \zeta_{2}\right)>0$ does not depend on the set $A$.

Now let us prove two theorems dealing with inequalities of harmonic functions analogous to inequality (1).

Th e o r e 2 . Let $D$ be a bounded domain of the 3-dimensional space with a sufficiently smooth boundary and $u(x)$ a harmonic function regular in $D$ and continuous with its first derivatives in the closed domain $\bar{D} ; x$ is a vector with components $x_{j}, j=1,2,3$.

Let $\varphi\left(\zeta_{1}, \zeta_{2}\right)$ be a vector-function depending on two complex arguments, and let its component functions $\varphi_{j}\left(\zeta_{1}, \zeta_{2}\right)(j=1,2,3)$ be analytic in the bicylinder of Theorem 1, continuous in the closed domain, real on the real plane, and suppose that the vectors

$$
\frac{\partial}{\partial \xi_{1}} \varphi\left(\xi_{1}, \xi_{2}\right), \quad \frac{\partial}{\partial \xi_{2}} \varphi\left(\xi_{1}, \xi_{2}\right)
$$

are nowhere collinear.

Let $S$ be a surface, defined by the equation

$$
x=\varphi\left(\xi_{1}, \xi_{2}\right),
$$

which lies strictly inside $D$, and let $A$ be a set of positive Lebesgue measure $\mu(A)$ in the disk

$$
\xi_{1}^{2}+\xi_{2}^{2} \leqq r^{2}<R^{2}
$$

and $B$ the image of $A$ under the mapping (3). 
Then, if the gradient of the function $u(x)$ satisfies the inequalities

$$
\begin{aligned}
& |\operatorname{grad} u(x)| \leqq \varepsilon, \quad x \in B, \\
& |\operatorname{grad} u(x)| \leqq M, \quad x \in \bar{D},
\end{aligned}
$$

it also satisfies the inequality

$$
|\operatorname{grad} u| \leqq M^{1-\omega(x)} \cdot \varepsilon^{\omega(x)},
$$

where $\omega(x)$ does not depend on $M, \varepsilon$ and satisfies the conditions

1. $0 \leqq \omega(x) \leqq 1, \quad x \in \bar{D}$,

2. $\omega(x)=1, \quad x \in B$,

3. $a(x) \mu(A) \leqq \omega(x), \quad x \in D$, where the function $a(x)>0$ does not depend on $A$.

Proof. It is known that the functions $\partial u(x) / \partial x_{j}$ are analytic in the real variables $x_{j}$ inside $D$ and may be extended into a cylindrical domain $\tilde{D}$ of the complex 3-dimensional space, $\tilde{D}=\{z=x+i y \mid x \in D\}$.

Let us denote these analytic extensions by $v_{j}(z)$ and consider the functions

$$
f_{j}\left(\zeta_{1}, \zeta_{2}\right)=v_{j}\left(\varphi\left(\zeta_{1}, \zeta_{2}\right)\right) .
$$

Evidently the functions $f_{j}\left(\zeta_{1}, \zeta_{2}\right)$ satisfy the conditions of Theorem 2 in a polycylinder

$$
\xi_{1}^{2}+\xi_{2}^{2}<R^{2}, \quad\left|\eta_{j}\right|<h,
$$

with a sufficiently small $h$. Therefore, on the surface $S$ the gradient of $u(x)$ satisfies the inequality

$$
|\operatorname{grad} u| \leqq M_{1}^{1-\omega(x)} \cdot \varepsilon^{\omega(x)}, \quad x \in S,
$$

where $\omega(x)$ has the properties

1. $0 \leqq \omega(x) \leqq 1, \quad x \in S$,

2. $\omega(x)=1, \quad x \in B$,

3. $a(x) \mu(A) \leqq \omega(x), \quad x \in S$.

Here $a(x)>0$ does not depend on $A$, and

$$
M_{1}=\left(\sum_{j=1}^{3} \sup \left|f_{j}\left(\zeta_{1}, \zeta_{2}\right)\right|^{2}\right)^{1 / 2} .
$$

From the maximum principle for the modules of the gradients of a harmonic function it follows that the constant $M_{1}$ in the inequality (6) may be replaced by $M$.

The theorem now follows from (6) and from the inequalities which characterize the conditional stability of the Cauchy problem for the Laplace equation (see [2], [3]). 
Th e o r e m 3. Let the surface $S$, satisfying the conditions of Theorem 2, bound a domain $D_{1} \subset D$. If the function $u(x)$ satisfies the inequalities

$$
\begin{array}{ll}
|u(x)| \leqq \varepsilon, & x \in B, \\
|u(x)| \leqq M, & x \in D,
\end{array}
$$

then the following inequality holds

$$
|u(x)| \leqq M^{1-\omega(x)} \cdot \varepsilon^{\omega(x)},
$$

where $\omega(x)$ is the function of Theorem 2.

Proof. From Theorem 2 it follows that the function $u(x)$ satisfies the inequality

$$
|u(x)|=M^{1-\omega(x)} \cdot \varepsilon^{\omega(x)}, \quad x \in S .
$$

By virtue of the maximum principle, inequality (7) holds also for all $x \in D_{1}$. The proof of the theorem follows from (7) and (1).

\section{References}

[1] Goluzin, G. M., and V. I. KryLov [Г. М. Голузин and В. И. Крылов]: Обобщение формулы Карлемана. - Mat. Sb. 40, 1933, 144-149.

[2] LANDIS, E. M. [Е. М. Ландис]: О некоторых свойствах решений эллиптических уравнений. - Dokl. Akad. Nauk SSSR 107:4, 1956, 640-643, and Uspehi Mat. Nauk 11:2 (68), 1956, $235-237$.

[3] LAVRENTIEV, M. M. [M. М. Лаврентьев]: О задаче Коши для уравнения Лапласа. - Izv. Akad. Nauk SSSR Ser. Mat. 20, 1956, 819-842.

[4] -»- О некоторых некорректных задачах математической физики. - Izdat. Sibirsk. Otdel. Akad. Nauk SSSR, Novosibirsk, 1962, 1-92.

Academy of Sciences

Department of Mathematics

117901 Moscow B-71

USSR

Received 26 May 1975
Academy of Sciences

Siberian Branch

Computing Center

Novosibirsk 630090

USSR 